\title{
Avaliação de tubo valvulado de pericárdio bovino em um modelo experimental animal
}

\author{
José Luiz Verde dos SANTOS*, Domingo M. BRAILE*, Marcelo José F. SOARES*, Walter RADE*, Marcos \\ Antônio ROSSI*, Rubens THEVENARD*, Dorotéia R. Silva SOUZA*
}

RBCCV 44205-103

SANTOS, J. L. V.; BRAILE, D. M.; SOARES, M. J. F.; RADE, W.; ROSSI, M. A.; THEVENARD, R.; SOUZA, D. R. S. - Avaliaçăo de tubo valvulado de pericárdio bovino em um modelo animal. Rev. Bras. Cir. Cardiovase., 5(1): 16-25, 1990.

RESUMO: Doenças congênitas, como a atresia pulmonar e a tétrade de Fallot, entre outras, têm sido corrigidas com o uso de condutos extracardiacos. Existe uma variedade de condutos, valvulados ou năo, que possibilita o acesso do fluxo sangūíneo do ventrículo direito à circulaçăo pulmonar. Contudo, os tubos plásticos com válvulas biológicas ou metálicas apresentam problemas de degeneraçăo e obstrução da prótese e peeling do tubo, além do alto custo. A experiência adquirida com o pericárdio bovino tratado, mostrando-se impermeável e de fácil sutura, possibilitou a confecção de tubos valvulados que, neste estudo, foram avaliados quanto a obstruçăo, calcificaçăo e dilataçăo. A utilizaçăo de um modelo experimental animal permitiu a avaliaçăo periódica detalhada por ecodopplercardiografia e cateterismo durante um, três e seis meses de evoluçăo. As peças recuperadas foram estudadas por microscopia óptica e raios $\mathrm{X}$, com a análise de diversas regiōes da bioprótese. O exame macroscópico mostrou o pericárdio dos tubos preservados com sinais de pseudo-endotelizaçăo, e a maioria das válvulas com calcificação moderada, mas ainda com boa funçăo. Săo apresentados e discutidos a técnica operatória empregada sem circulaçăo extracorpórea e os resultados com o seguimento de cinco animais (carneiros), durante seis meses. Os autores concluem que o enxerto tubular valvulado de pericárdio bovino mostrou bons resultados na via de saida do ventrículo direito, permitindo prever um resultado satisfatório quando empregado em seres humanos, pela conhecida relaçăo de degeneraçáo acelerada das próteses biológicas no modelo experimental estudado, muitas vezes maior que no homem.

DESCRITORES: tubo valvulado, biológico.

\section{INTRODUÇĀO}

Os condutos extracardiacos vêm sendo usados desde a década de 60 , com os primeiros implantes realizados com sucesso por RASTELLI et alii (1965) ${ }^{34}$, utilizando tubo năo valvulado de pericárdio autógeno, e por ROSS \& SOMERVILLE (1966) ${ }^{35}$, com enxerto homólogo de aorta ascendente e valva aórtica, ambos em atresia pulmonar.

Posteriormente, a utilizaçăo de condutos foi extendida à correção de outras cardiopatias, tais como aneu- rismas da aorta ascendente com ou sem insuficiência valvar ${ }^{3}$, 19. 24 , hipoplasia congênita atrioventricular ${ }^{28}$, transposição das grandes artérias, comunicação interventricular e estenose pulmonar ${ }^{31}$, ectasia ânulo-aórtica ${ }^{27}$, obstruçāo da via de saída do ventrículo esquerdo ${ }^{5}$, entre outras.

Os resultados com condutos extracardiacos estimularam o aparecimento de uma variedade de condutos sintéticos e biológicos, valvulados ou não. Os biológicos apresentam-se de dois tipos, homólogos aórticos e heterólogos. Entre os enxertos heterólogos, incluem-se os

Trabalho realizado no Instituto de Moléstias Cardiovasculares de Săo José do Rio Preto, SP, Brasil.

Apresentado ao 17: Congresso Nacional de Cirurgia Cardiaca. Belo Horizonte, MG, 6 e 7 de abril, 1990.

- Do Instituto de Moléstias Cardiovasculares de São José do Rio Preto.

Endereço para separatas: José Luiz Verde dos Santos. Rua Castelo D’Água, 3030. 15015 Săo José do Rio Preto, SP, Brasil. 
SANTOS, J. L. V.; BRAILE, D. M.; SOARES, M. J. F.; RADE, W.; ROSSI, M. A.; THEVENARD, R.; SOUZA, D. R. S. - Avaliaçăo de tubo valvulado de pericárdio bovino em um modelo experimental animal. Rev. Bras. Cir. Cardiovasc., 5(1): 16-25, 1990.

com tubo sintético e válvula biológica, comumente a porcina ${ }^{33}$, e aqueles com tubo de tecido biológico e válvula biológica sem suporte ${ }^{20,31}$.

Entretanto, mesmo diante dos vários tipos de condutos extracardíacos, devido às limitaçōes e às complicaçōes inerentes, as pesquisas permanecem incessantes. Quanto aos homoenxertos aórticos, ainda se discute sua difícil obtenção e conservação, além da alta incidência de calcificação e obstrução ${ }^{29}, 30,37$. Em contrapartida, SCHLICHTER \& KREUTZER (1985) ${ }^{38}$ lembram sua potencialidade de crescimento de tubos confeccionados com pericárdio autógeno. Os heteroenxertos com tubo sintético também apresentam problemas de obstrução ${ }^{4}$. 6. 16. 17 e peeling do tubo; além disso, são onerosos. Os heteroenxertos com tubo de tecido biológico e válvula biológica sem suporte parecem satisfatórios; no entanto, necessitam de um maior acompanhamento, para ulterior análise ${ }^{31}$.

O pericárdio bovino com tratamento especial é um dos tecidos biológicos mais amplamente explorados em cirurgia, como nos casos de fechamento de comunicação interventricular, na comunicação interatrial, na ampliaçăo da via de saída de ventrículo direito na tétrade de Fallot, no reparo de lesōes diafragmáticas, no fechamento do pericárdio após cirurgia, no apoio de suturas da aorta, no alargamento da aorta e em istmoplastia na coarctação da aorta ${ }^{3,8,15}$.

A experiência do Serviço IMC com o pericárdio bovino tratado, mostrando-se impermeável e de fácil sutura, estimulou os autores a confeccionarem um tubo de pericárdio com uma válvula também de pericárdio bovino montada em suporte.

O presente estudo objetivou a avaliação desse tubo valvulado quanto à obstrução, à calcificação e à dilataçăo, utilizando-se, para isso, um modelo experimental animal, o que permitiu a realizaçăo de exames periódicos, de acordo com a conveniência do estudo.

\section{CASUÍSTICA E MÉTODOS}

\section{Preparaçâo do Pericárdio e Confeçâao da Bioprótese}

O pericárdio bovino é coletado em frigoríficos, imediatamente após o abate dos animais, e transportado em soluçāo hipertónica de $\mathrm{NaCl}$ a pH 7,4 a $4^{\circ} \mathrm{C}$. No laboratório, após a limpeza nessa soluçăo, o pericárdio é montado em suporte e submetido a fixaçăo em solução de glutaraldeído a $0,5 \%$ por 15 dias, a $4^{\circ} \mathrm{C}$ e, durante três dias, à temperatura ambiente, com trocas periódicas da solução. Completando o período de fixação, o pericárdio é colocado em solução de formaldeído a $4 \%$ para conservação. Nessa etapa, sáo realizados os testes de controle de qualidade, entre eles, encolhimento, elongação, rotura, tensão superficial crítica, medida de espes- sura e histologia. São liberados apenas os pericárdios que preencherem os critérios previamente estabelecidos. Esses testes permitem detectar a atuação adequada do glutaraldeído no curtimento do material, que deve manter o alinhamento dos feixes de fibras colágenas sem alterar sua ondulação natural, proporcionando a devida elasticidade mecânica ao tecido.

\section{Confecção da Válvula}

Assim processado, o pericárdio é empregado na confecção da válvula e do tubo. A válvula compreende, basicamente, um suporte de Delrin com aro de açō inoxidável, inicialmente coberto por Dacron 1642 , com fixação ulterior de um cone de pericárdio sobre ele, visando à moldagem dos seus componentes. Após a montagem, a bioprótese é submetida a uma avaliação final, quando são analisados o aspecto geral, a coaptaçăo dos seus componentes, o acabamento das hastes, a ausência de rugas, a flexibilidade, a elasticidade e a sua funcionalidade. Além disso, é revista a ficha de registro que acompanha cada bioprótese, incluindo a data da coleta do pericárdio, os lotes das soluçóes, o responsável pela sua confecçăo e os resultados dos testes de controle de qualidade. Nos testes finais, incluem-se a bacteriologia e o certificado dimensional pós-embalagem, realizado por meio de radiografias. Durante a fase de confecção, as biopróteses são conservadas em formaldeído a $4 \%$.

\section{Confeçăa do Tubo}

O diâmetro da válvula determina a largura do patch de pericárdio bovino, que apresenta até $20 \mathrm{~cm}$ de comprimento. A válvula de pericárdio já confeccionada é, entăo, suturada nesse retalho, distando $2 / 3$ de uma extremidade do tubo e 1/3 da outra. Posteriormente, o patch é fechado com pontos individuais, adquirindo, assim, a forma de tubo valvulado.

\section{Amostra Estudada}

Foram estudadas cinco ovelhas pesando de $28 \mathrm{~kg}$ a $35 \mathrm{~kg}(\mathrm{M}=32,8 \mathrm{~kg})$ submetidas a cirurgia com tubo valvulado de pericárdio bovino na via de saída do ventrículo direito, com seguimento de seis meses. O controle dos animais foi feito mensalmente por ecodoppler e por cateterismo aos três meses de implante. As peças resgatadas foram analisadas macroscópica e radiograficamente com senógrafo de Thompson - CGR ( 35 a 39 KV) e enviadas amostras das regiōes proximal e distal 
SANTOS, J. L. V.; BRAILE, D. M.; SOARES, M. J. F.; RADE, W.; ROSSI, M. A.; THEVENARD, R.; SOUZA, D. R. S. - Avaliação de tubo valvulado de pericárdio bovino em um modelo experimental animal. Rev. Bras. Cir. Cardiovasc., 5(1): 16-25, 1990.

dos tubos, bem como de várias áreas dos componentes da válvula, para o estudo histológico.

\section{Técnica Cinúrgica}

Após induçăo anestésica, foi feita monitorizaçăo da pressão arterial (PA) por dissecção da artéria femoral direita, da pressão venosa central (PVC) por dissecção da veia femoral direita, e do eletrocardiograma (ECG) por fixação dos eletrodos com pontos em regiōes tricotomizadas previamente. $\mathrm{O}$ animal foi colocado em decúbito lateral direito, feita tricotomia, assepsia com povidine tintura e colocação de campos cirúrgicos. A abordagem do mediastino foi feita através de toracotomia esquerda no quarto espaço intercostal. O pericárdio foi aberto e fixado aos campos. Foram colocados $20 \mathrm{ml}$ de cloridrato de lidocaína a $2 \%$ no saco pericárdico, para evitar arritmias na fase de pinçamento da via de saída do ventrículo direito. $\mathrm{O}$ tubo valvulado de pericárdio bovino com 21 $\mathrm{cm}$ ou $23 \mathrm{~cm}$ foi lavado em $3000 \mathrm{ml}$ de soro fisiológico. O tubo foi medido e cortado em bizel, tomando-se o cuidado de deixar a prótese o mais próximo possível do tronco pulmonar. Um ponto de polipropilene Ethicon 4-0 fo passado na parede ventricular na via de saída do ventrículo direito, para tracioná-la. O pinçamento amplo foi realizado com pinça DeBakey e as condiçōes hemodinâmicas foram analisadas. Quando houve qualquer alteraçāo, como hipotensāo, arritmia cardiaca, ou distensão do ventrículo direito, a via de saída foi despinçada e novo procedimento foi realizado, após alguns minutos. Em boas condiçōes hemodinâmicas, a região foi incisada com bisturi e ampliada com tesoura de Potz, para que a anastomose proximal ficasse grande o suficiente para não causar estenose. Procedeu-se à confecção da mesma com polipropilene 5-0 através de sutura contínua (Figuras 1 e 2). O despinçamento foi realizado e o comprimento do tubo foi confirmado. Durante a estabilização hemodinâmica, o tronco pulmonar foi laçado com fio de algodāo que, posteriormente, serviu para sua ligadura. O ligamento arterioso foi dissecado e ligado, já que a anastomose distal foi feita no tronco pulmonar em direção à artéria pulmonar esquerda. Um novo ponto de polipropilene Ethicon 4-0 foi passado, desta vez na parede do tronco pulmonar para sua tração e pinçamento com a mesma pinça DeBakey. Novamente as condiçōes hemodinâmicas foram analisadas, feita abertura do tronco com bisturi e ampliada a incisăo com tesoura de Potz. A anastomos distal foi confeccionada com polipropilene Ethicon 5-0, sutura contínua. Com o despinçamento, foi revista a hemostasia da linha de sutura, o tronco pulmonar foi ligado, desviando o sangue para o enxerto tubular valvulado. Após o fechamento por planos, o animal foi recuperado. Os cateteres foram retirados e feita extubação após a retirada do dreno torácico. Em seguida, o animal foi mantido em biotério, onde permaneceu por 48 horas em observação. Após esse período, foi transportado para local aberto, dispondo de pastagem e ração balanceada.

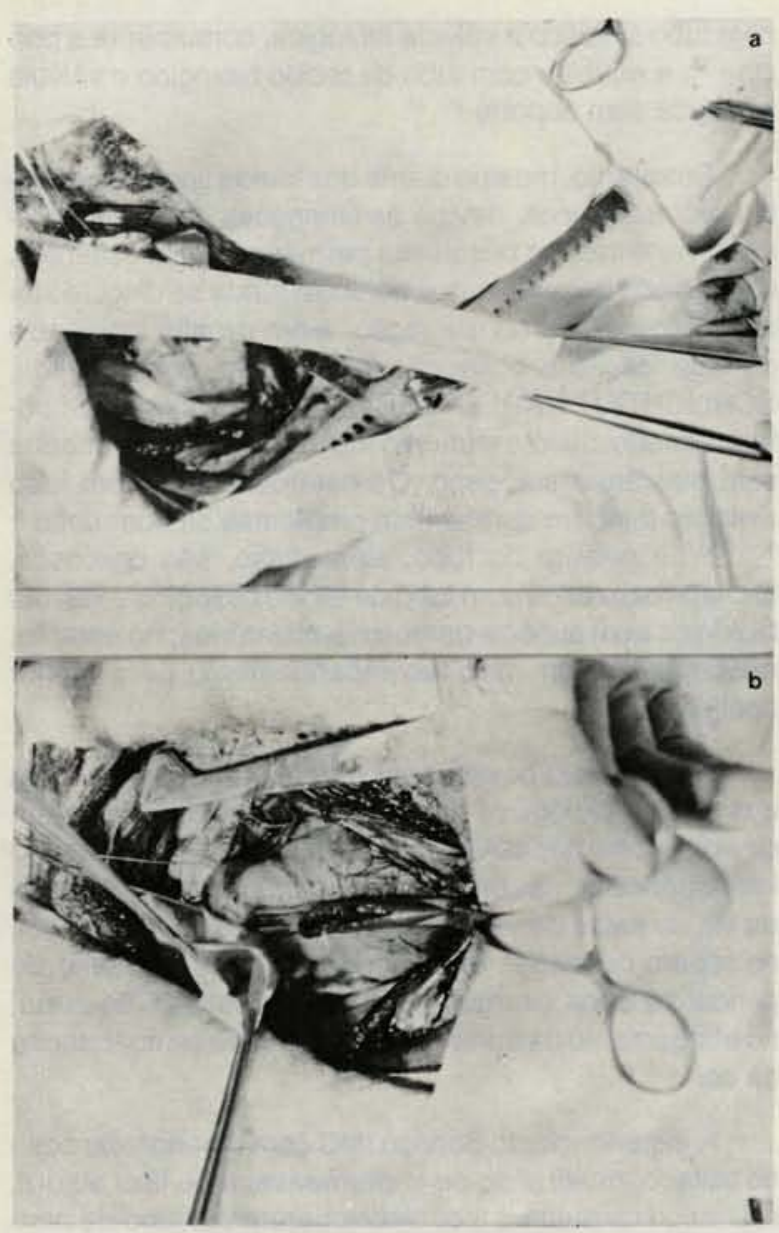

Fig. 1 - (a) Tubo valvulado de pericárdio bovino mostrando a sutura da prótese na região mediana; (b) pinçamento da via de saida do ventriculo direito, abertura e realizaçăo da anastomose proximal com sutura contínua de polipropilene Ethicon 5-0.

\section{RESULTADOS}

Todos os animais completaram seis meses de acompanhamento, conforme protocolo. Uma das oveIhas, mesmo em fase de gestação, apresentou comportamento normal, parindo dois conceptos sadios. Os resultados dos exames ecodopplercardiográficos evidenciaram gradientes entre 15 e $57 \mathrm{mmHg}$ imediatamente antes do sacrifício (Tabela 1), com o maior gradiente apresentado pela ovelha que passou pela fase de gestaçăo com o implante. A análise da peça revelou estenose na anastomose proximal, explicando tal gradiente. O tubo e a válvula ficaram protegidos de alteraçōes, como fibrose e calcificaçăo, pela brusca diminuição da prssăo ao nível da anastomose.

O cateterismo realizado três meses após o implante revelou o tubo pérvio e ausência de calcificaçăo (Figura 3). O gradiente transvalvular foi medido, variando de 15 a 25 mmHg (Figuras 4 e 5 ). 
SANTOS, J. L. V.; BRAILE, D. M.; SOARES, M. J. F.; RADE, W.; ROSSI, M. A.; THEVENARD, R.; SOUZA, D. R. S. - Avaliaçāo de tubo valvulado de pericárdio bovino em um modelo experimental animal. Rev. Bras. Cir. Cardiovasc., 5(1): 16-25, 1990.

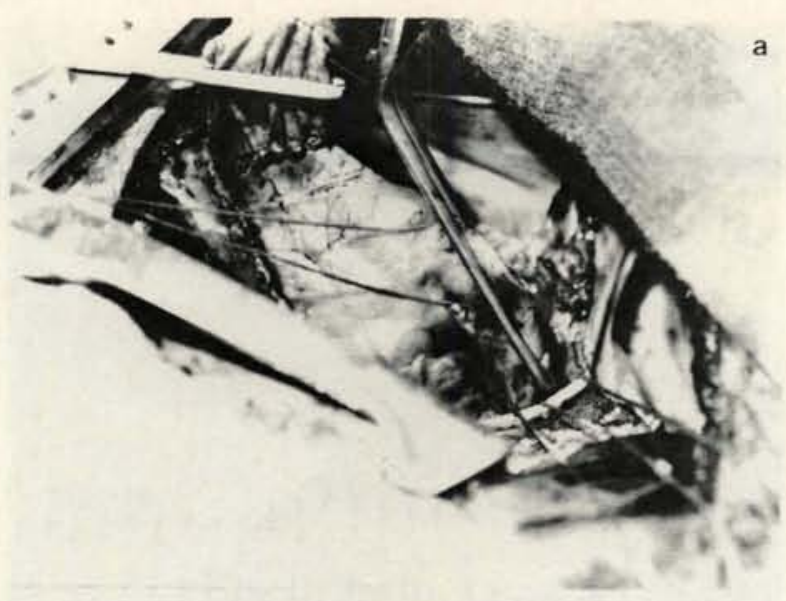

: calcificação, mostraram-se flexíveis. Tal mineraliza1o foi evidenciada radiograficamente (Figura 6). Não uve detecção de trombos nas peças recuperadas.

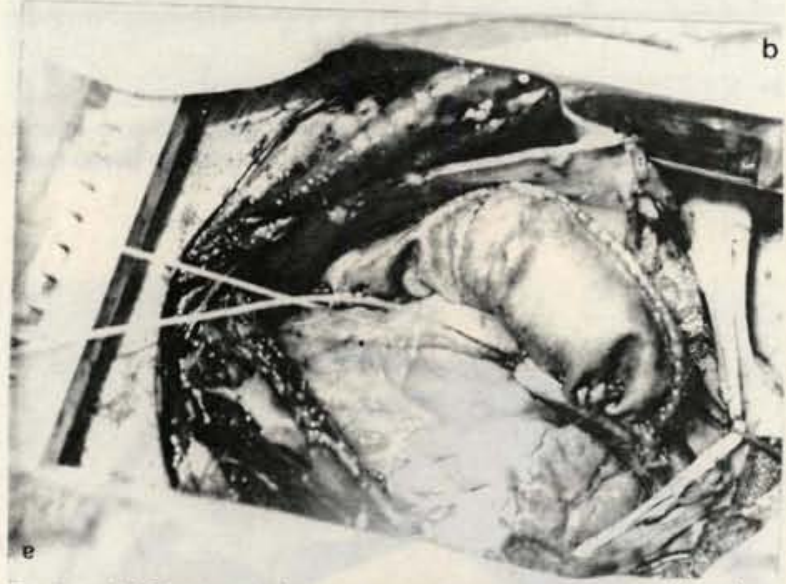

Fig. 2 - (a) Pinçamento do tronco pulmonar em aıreçao a artéria pulmonar esquerda com pinça DeBakey; (b) anastomoses conclufdas; oclusăo do tronco pulmonar com cadarço.

TABELA 1

RESULTADOS ECODOPPLERCARDIOGRAFICOS DOS DOIS E SEIS MESES DE IMPLANTE DO TUBO VAL VULADO DE PERICÁRDIO BOVINO NA VIA DE SAIDA DO VENTRICULO DIREITO

\begin{tabular}{cccc}
\hline \multirow{2}{*}{ Animal } & Peso $(\mathrm{Kg})$ & \multicolumn{2}{c}{ Gradientes Ecodoppler $(\mathrm{mmHg})$} \\
\cline { 3 - 4 } & & 3 meses & 6 meses \\
\hline 82 & 34 & 21 & 57 \\
83 & 34 & 18 & 35 \\
85 & 28 & 23 & 29 \\
86 & 35 & 10 & 15 \\
47 & 33 & 8 & 28 \\
\hline
\end{tabular}

$\mathrm{Na}$ recuperação dos tubos, estes apresentavam bom aspecto, superfície lisa com aderência moderada e pequena deposiçăo de cálcio nos elementos valvulares, sendo rinais intensa no anel e nas comissuras (Tabela 2). Os componentes da válvula, mesmo com pontos

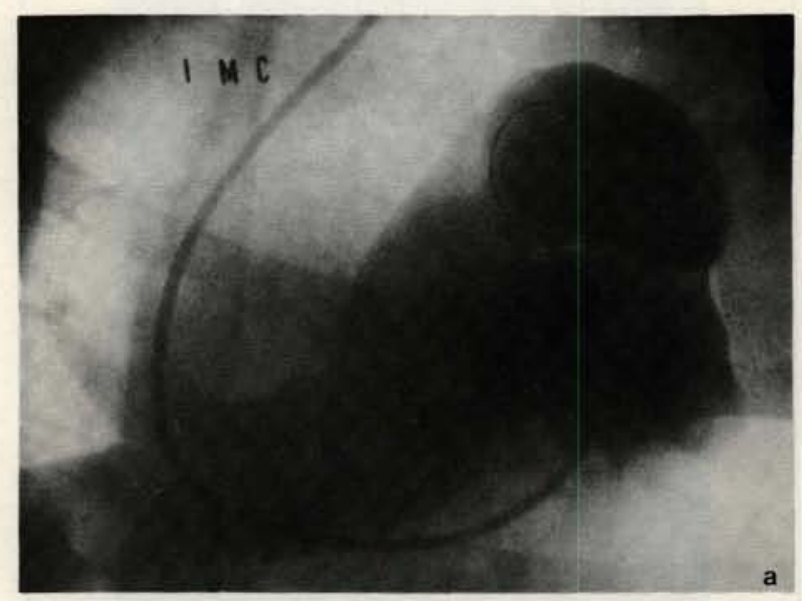

TABELA 2

SPECTOS MACROSCOPICOS DOS TUBOS VALVULADOS EPERICARDIO BOVINO APOS SEIS MESES DE IMPLANTE NA VIA DE SAIDA DO VENTRICULO ESQUERDO

\begin{tabular}{ccl}
\hline Animal & Peso $(K g)$ & \multicolumn{1}{c}{ Aspecto macroscópico } \\
\hline 82 & 34 & Estenose na anastomose proximal \\
83 & 34 & Prótese c/calcificação moderada \\
85 & 28 & Prótese fibrosada e c/calcificação discreta \\
85 & 35 & Prótese com calcificação discreta \\
87 & 33 & Prótese com calcificação discreta \\
\hline
\end{tabular}

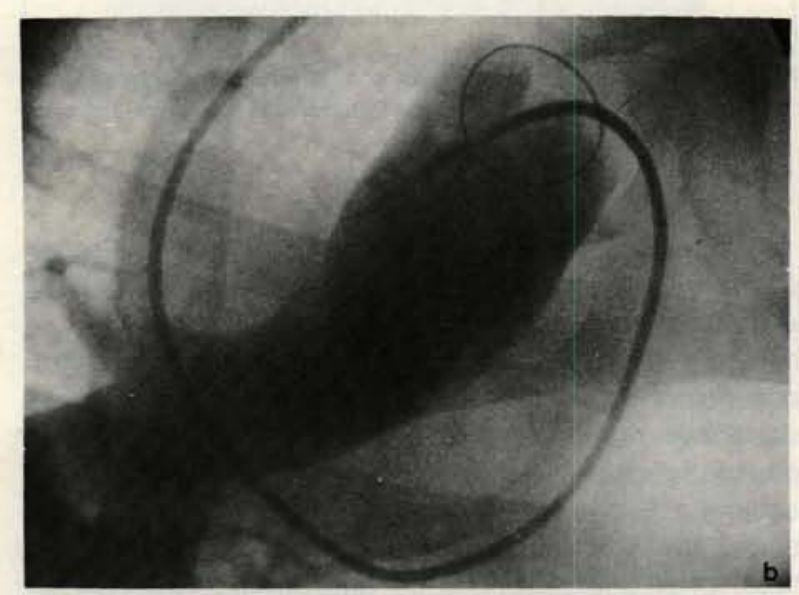

Fig. 3 - Ventriculografia direita mostrando local da anastomose proximal e plano valvar (anel); (b) injeçăo na regiáo do tubo valvulado revelando total suficiência da prótese e ausência de calcificaçăo visível. 
SANTOS, J. L. V.; BRAILE, D. M.; SOARES, M. J. F.; RADE, W.; ROSSI, M. A.; THEVENARD, R.; SOUZA, D. R. S. - Avaliação de tubo valvulado de pericárdio bovino em um modelo experimental animal. Rev. Bras. Cir. Cardiovasc., 5(1): 16-25, 1990.

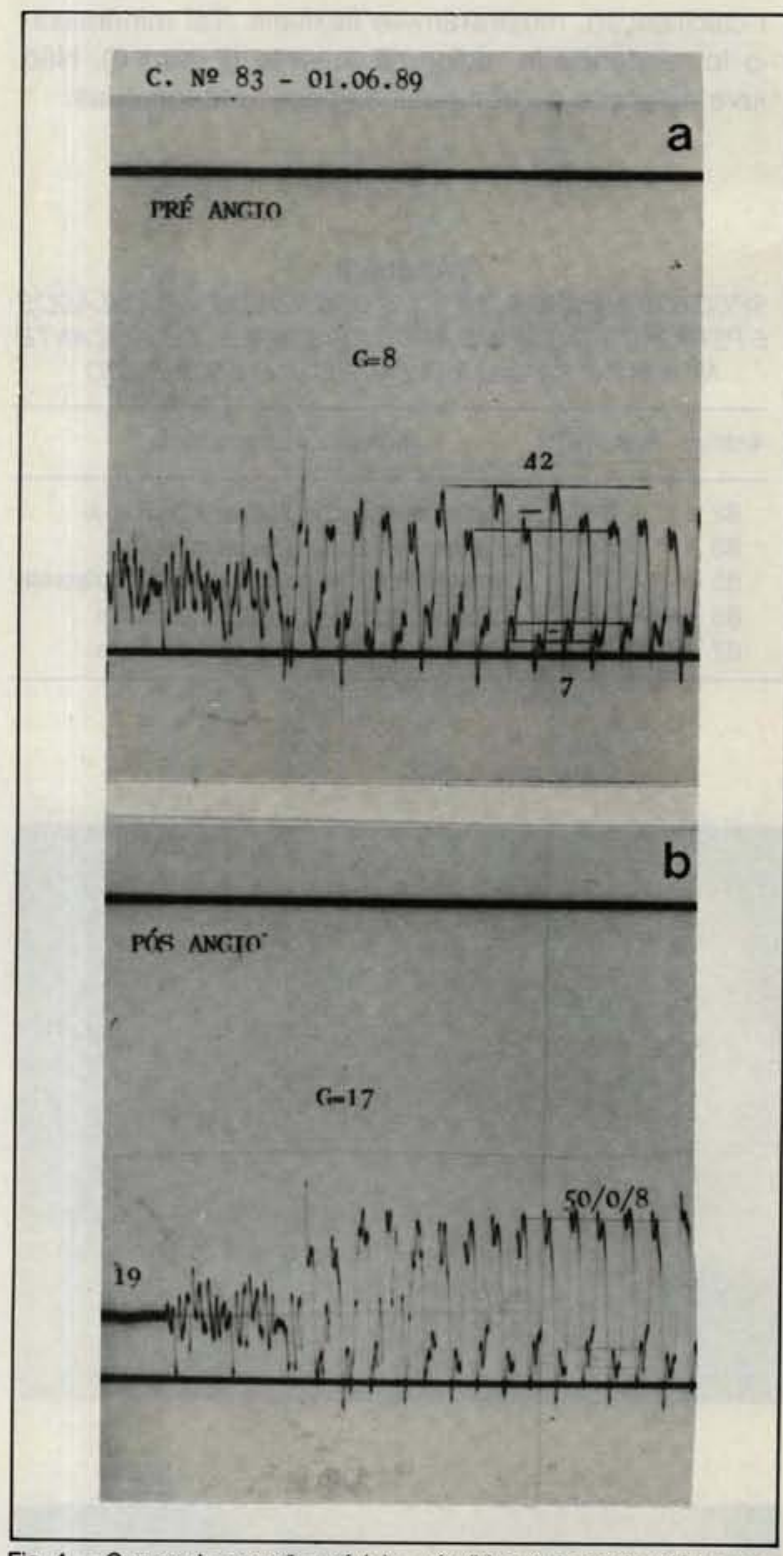

Fig. 4 - Curvas de pressão pré (a) e pós (b) estudo angiográfico mostrando aumento do gradiente transvalvular de 8 para $17 \mathrm{mmHg}$.

O estudo histológico das várias regiōes dos tubos e das válvulas revelou que as superfícies da válvula e do tubo apresentavam-se rugosas e constituídas de colágeno próprio do pericárdio bovino. Foram registrados, no pericárdio da válvula e do tubo, discreta alteração das fibras colágenas e focos microscópicos de depósitos difusos de cálcio, sem modificar, contudo, a arquitetura colágena. Essas alteraçōes patológicas, embora qualitativamente idênticas para o pericárdio da válvula e do tubo, foram quantitativamente mais pronunciadas na válvula. As alterações observadas não comprometeram, de maneira significativa, a integridade estrutural do pericárdio bovino.

\section{c. №86 01.06 .89}

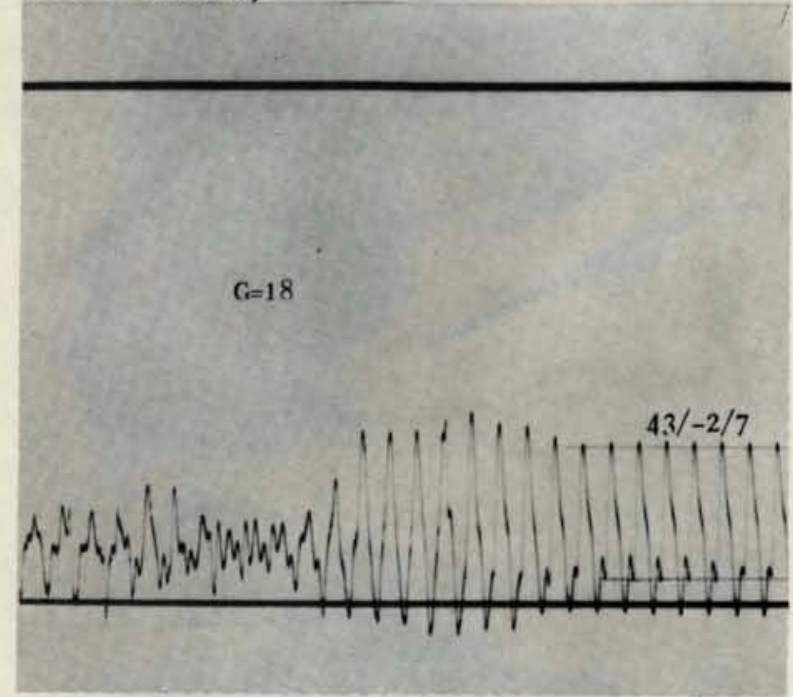

Fig. 5 - Curva de pressão obtida pós estudo angiográfico; o cateter é recuado do tronco pulmonar para o ventrículo direito mostrando gradiente de $18 \mathrm{mmHg}$.

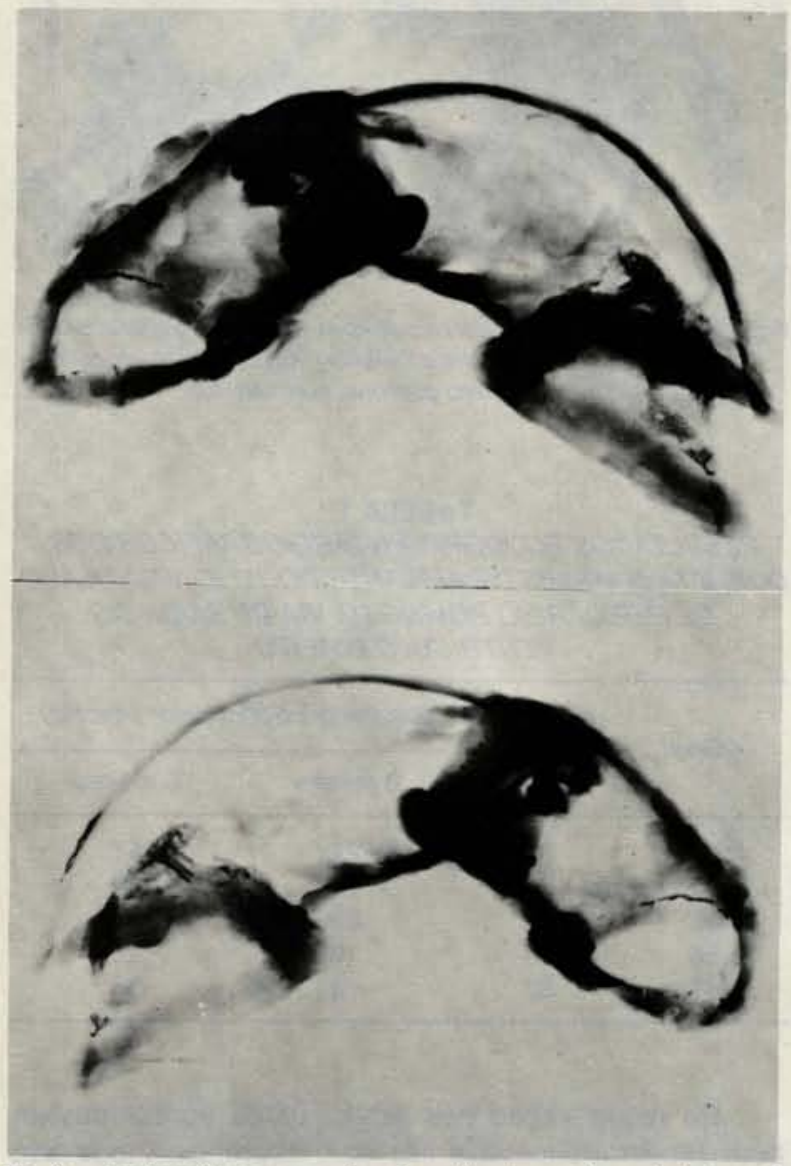

Fig. 6 - Radiografia com mamógrafo realizada no tubo valvulado pos morte mostrando discreta calcificaçăo da prótese valvular predominante nas hastes năo comprometendo a boa abertura da mesma. 
SANTOS, J. L. V.; BRAILE, D. M.; SOARES, M. J. F.; RADE, W.; ROSSI, M. A.; THEVENARD, R.; SOUZA, D. R. S. - Avaliação de tubo valvulado de pericárdio bovino em um modelo experimental animal. Rev. Bras. Cir. Cardiovasc., 5(1): 16-25, 1990.

\section{DISCUSSĀO}

O carneiro mostrou-se eficaz como modelo experimental para esse estudo, permitindo 0 acompanhamento periódico, necessário para a avaliaçāo do tubo valvulado de pericárdio bovino, em curto prazo. Esse animal é de nossa preferência, por ser dócil, com cavidade torácica de tamanho razoável para manuseio sem dificuldades, com rápida fase de crescimento, permitindo, em curto periodo, 0 acompanhamento de todas as fases do desenvolvimento. Além disso, as alteraçōes detectadas em biopróteses valvulares neles implantadas são morfologicamente semelhantes àquelas observadas no homem, ocorrendo, contudo, com maior rapidez.

A cirurgia de implante do bioenxerto na via de saída do ventrículo direito foi de fácil execução, graças a alta flexibilidade, adaptabilidade e ausência de sangramento do bioenxerto, mesmo sem medidas de pré-coagulação, geralmente necessárias para outros tipos de condutos valvulados ${ }^{24}$.

O posicionamento do tubo valvulado e sua anastomose, como nesse estudo, tem sido preocupação geral, na tentativa de se evitar a angulação do enxerto, muito comum quando o tubo é maior que o necessário, causando obstrução na anastomose proximal ${ }^{7}$. Tal fato foi evidenciado no início desse estudo, na tentativa de padronização da técnica cirúrgica. A partir daí, a válvula, que antes ocupava o centro do tubo de pericárdio bovino, foi deslocada para a extremidade, resultando em um tubo com 2/3 livres na região proximal e 1/3 na distal.

A obstrução de condutos valvulados é comum na válvula e na anastomose distal, como consequeência de trombose, da formação intensa de neoíntima, do deslocamento da íntima, da calcificação do tubo e/ou da válvula, ou de problemas técnicos ${ }^{1,6}, 13,14,16,26$. Além dessas causas, a compressão do tubo valvulado entre o gradil costal e o coração pode acarretar, também, obstrução ${ }^{4}$. Esse tipo de complicaçăo nem sempre ocorre em apenas uma região, mas em vários níveis de um mesmo conduto ${ }^{21}$.

A obstrução é reconhecida em todos os tipos de condutos valvulados, inclusive em homoenxerto tubular valvulado preservado com antibiótico ". A baixa porosidade dos tubos de Dacron pode predispor à obstrução ${ }^{6}$, surgindo, como alternativa, os tubos de alta porosidade ${ }^{15}$. Contudo, esse tipo de conduto está sujeito a obstrução aguda ${ }^{6}$.

Há consideraçōes, ainda, sobre a área da válvula porcina que compóe o heteroenxerto valvulado, no caso, menor que a dos homoenxertos aórticos ${ }^{25}$. Por outro lado, a área valvular da bioprótese ora estudada não é preocupante, os resultados são satisfatórios em decorrência da própria técnica de montagem desse tipo de prótese ${ }^{5,9}$.
Nesses seis meses de acompanhamento, não foram registrados óbitos por obstruçăo da bioprótese. Os animais tiveram comportamento e vida normais, de tal modo que uma das ovelhas iniciou e concluiu uma gravidez durante a fase de estudo, sem, contudo, apresentar qualquer tipo de complicação. Nem mesmo outras complicaçōes não raras, como a deiscência, rompimento da anastomose em implantes de tubos valvulados com potencial letal ${ }^{27}$, foram evidenciadas nesse estudo. Contudo, em uma experiência inicial com o tubo valvulado de pericárdio bovino na recuperação das peças, ao contrário do presente estudo, evidenciou-se a presença de pequenos trombos na válvula, sugerindo a falta de domínio técnico desse tipo de cirurgia em tal ocasião. Com a padronização da técnica, os resultados foram-se aperfeiçoando, a ponto de não se detectarem trombos nas próteses recuperadas.

O uso da ecodopplercardiografia possibilitou o exame periódico dos animais de maneira não invasiva, visando determinar o grau progressivo de obstruçăo da via de saída do ventrículo direito. Nesse caso, foi registrada apenas uma alteração lenta no gradiente, basicamente por calcificação e fibrose progressiva da válvula, visível macroscopicamente na recuperação das peças. Os tubos de pericárdio apresentaram-se desobstruídos, sem fibrose intensa da neoíntima, observada em tubos de Dacron ${ }^{7}, 12$ e de politetrafluoretileno. Neste último, apesar de mais reduzida ${ }^{10}$, é freqüentemente a causa primária de obstrução do enxerto, do que propriamente a válvula ${ }^{11}$.

Esse resultado foi comprovado radiograficamente com o registro de mineralização mais freqüente no anel e comissuras, regiōes pouco críticas para o bom desempenho da bioprótese.

O estudo histológico da válvula e das diversas regiōes do tubo de pericárdio confirmou a presença de mineralização no tecido, principalmente da válvula e menos acentuadamente do tubo. Por outro lado, JONAS et alii $(1988)^{22}$, em estudo comparativo experimental de homoenxertos valvulados criopreservados e frescos, encontraram calcificação proeminente na parede do tubo de todos os animais, com as válvulas dos condutos geralmente permanecendo livres de calcificaçăo. Confirmaram ainda, a presença de trombos na maioria das válvulas criopreservadas.

Entre todas as complicaçōes que afetam as próteses de tecido biológico, a calcificaçăo é, sem dúvida, a mais preocupante, desencadeando uma série de estudos com vistas a novas técnicas de fixação, novos agentes de curtição e tratamentos específicos anticalcificação. Destacam-se, atualmente, os estudos com o sulfato de sódio dodecil (T6) ${ }^{2}, 23$, com os difosfonatos, etanohidroxidifosfonato (EHDP) ${ }^{25}$ e aminopropanohidroxidifosfonato (APDP) ${ }^{40}$, o fosfocitrato ${ }^{39}$, o éter sulfúrico acidificado, testado por RUSSI et alii ${ }^{36}$, que, atualmente, têm reali- 
SANTOS, J. L. V.: BRAILE, D. M.; SOARES, M. J. F.; RADE, W.; ROSSI, M. A.; THEVENARD, R.; SOUZA, D. R. S. - Avaliaçâo de tubo valvulado de pericárdio bovino em um modelo experimental animal. Rev. Bras. Cir. Cardiovasc., 5(1): 16-25, 1990.

zado testes também com difosfonatos. Uma outra proposta mais recente é o uso de acyl azide como fixador de tecido biológico, revelando resultados animadores ${ }^{32}$.

PRENGER et alii (1988) ${ }^{33}$ sustentaram que a administraçāo de acenocoumarol (warfarina sódica) no mínimo por três meses, em pacientes com implante de tubo de Dacron com válvula porcina, pareceu contribuir para resultados favoráveis por longo tempo.
Concluindo, as próprias complicaçōes diante de implantes de condutos valvulados sintéticos ou biológicos justificam novas tentativas em busca de uma prótese que exponha menos ao risco o paciente, reduzindo o índice de complicaçōes e reoperaçōes. O tubo valvulado de pericárdio bovino totalmente biológico mostrou bons resultados na via de saída do ventrículo direito de oveIhas, permitindo prever resultado satisfatório em pacientes, pela conhecida relação de degeneração acelerada das próteses biológicas no modelo experimental estudado, muitas vezes maior que o homem.

RBCCV 44205-103

SANTOS, J. L. V.; BRAILE, D. M.; SOARES, M. J. F.; RADE, W.; ROSSI, M. A.; THEVENARD, R.; SOUZA D. R. S. - Evaluation of bovine pericardium valved conduit in an animal experimental model. Rev. Bras. Cir. Cardiovasc., 5(1): 16-25, 1990.

ABSTRACT: Congenital diseases as pulmonary atresia, tetralogy of Fallot, among others, have been corrected using the extracardiac conduits. There is a variety of conduits valved or not that make possible the access of sanguineous flux from right ventricle to pulmonary circulation. However, the synthetic conduits with biologic or metalic valves shows prosthesis degeneration and obstruction problems and peeling of the conduit, moreover, they are very expensive. The experience with treated bovine pericardium showing it is impermeable and easy to suture, led to the production of valved conduits that in this study were evaluated for obstruction, calcification and stretching. The utilization of an animal experimental model allowed the detailed periodic evaluation by echodopplercardiography and cateterism during one, three and six months. The recuperated conduits were studied by optical microscopy and $X$ ray, with several parts of the bioprosthesis analysed. The microscopic examination showed the conduits pericardium preserved with signs of pseudo-endotelization, and the majority of valves with moderate calcification, but with good function yet. The surgery technique without extracorporeal circulation and the results with the follow-up of five animals during six months are presented and discussed. The authors conclude that the bovine pericardium valved conduit showed good results in the right ventricule outlet, allowing to expect a satisfatory result when applied in human beings by the known relation of acelerated degeneration of the biological prostheses on the studied experimental model, several times greater than in man.

DESCRIPTORS: valvar tube graft, biologic.

\section{REFERÊNCIAS BIBLIOGRÁFICAS}

1 AGARWALL, K. C.; EDWARDS, W. D.; FELDT, R. H.; DANIELSON, G. K.; PUGA, F. J.; McGOON, D. C. - Clinicopathological correlates of obstructed righ-sided porcine-valved extracardiac conduits. J. Thorac. Surg., 81: 591-601, 1981.

2 ARBUSTINI, E.; JONES, M.; MOISES, R. D.; EIDBO, E. E.; CARROL, R. J.; FERRANS, V. J. - Modification by the Hancock T6 process of calcification of bioprosthetic cardiac valves implanted in sheep. $\mathrm{Am}$. J. Cardiol., 53: 1388-1396, 1984.

3 ARDITO, R. V.; SANTOS, J. L. V.; MAYORQUIM, R. C.; GRECO, O. T.; ZAIANTCHICK, M.; SOTO, H. G.; JACOB, J. L. B.; BRAILE, D. M. - Substituiçāo completa da aorta ascendente e da valva aórtica com tubo valvulado de pericárdio bovino. Rev. Bras. Cir. Cardiovasc., 2: 129-138, 1987.
4 BAILEY, W. W.; KIRKLIN, J. W.; BARGERON, L. M.; PACIFICO, A. D.; KOUCHOUKOS, N. T. - Late results with conduits from venous ventricle to pulmonary arteries. Circulation, 56: (Parte 2): 73-79, 1977.

5 BEHRENDT, D. M. \& ROCCHINI, A. - Relief of left ventricular outflow tract obstruction in infants and small children with valved extracardiac conduits. Ann. Thorac. Surg., 43: 82-86, 1987.

6 BEN-SHACHAR, G.; NICOLOFF, D. M.; EDWARDS, J. E. - Separation of neointima from Dacron graft causing obstruction. J. Thorac. Cardiovasc. Surg., 82: 268-271, 1981.

7 BOYCE, S. W.; TURLEY, K.; YEE, E. S.; VERRIER, E. D.; EBERT, P. A. - The fate of the $12 \mathrm{~mm}$ porcine valved conduit from the right ventricle to the pulmonary artery. J. Thorac. Cardiovasc. Surg., 95: 201-207, 1988. 
SANTOS, J. L. V.; BRAILE, D. M.; SOARES, M. J. F.; RADE, W.; ROSSI, M. A.; THEVENARD, R.; SOUZA, D. R. S. - Avaliação de tubo valvulado de pericárdio bovino em um modelo experimental animal. Rev. Bras. Cir. Cardiovasc., 5(1): 16-25, 1990.

8 BRAILE, D. M.; ARDITO, R. V.; GRECO, O. T. - Alargamento da raiz da aorta com "patch" de pericárdio bovino preservado pelo glutaraldeído. Arq. Bras. Cardiol., 41: $289-296,1983$.

9 BRICK, A. V.; MIANA, A. A.; COLEN, E. A.; PASSOS, P. H. C.; BORGES, A.; JORGE, P. C.; BRAILE, D. M.; GRECO, O. T.; ARDITO, R. V.; SANTOS, J. L. V.; MAYORQUIM, R. C.; LIMA, E. R.; ZAIANTCHICK, M.; CAMPOS, K. L.; GOLLARZA, H. S.; SOUZA, R. S. D.; BROFMAN, P. R.; LOURES, D. R. R.; CARVALHO, R. G.; RIBEIRO, E. J. - Seguimento de 9 anos da bioprótese valvar cardiaca de pericárdio bovino IMC-Biomédica: estudo multicêntrico. Rev. Bras. Cir. Cardiovasc., 2: 189-199, 1987.

10 BROWN, J. W.; HALPIN, M. P.; RESCORLA, F. J.; VANNATTA, B. W.; FIORE, A. C.; SHIPLEY, G. D.; BIZUNCH, M.; BILLS, R.; WALLER, B. - Externally stended polytetrafluoroethylene valved conduits for right heart reconstruction and experimental comparison with Dacron valved conduits. J. Thorac. Cardiovasc. Surg., 90: 833-841, 1985.

11 BULL, C.; MCARTNEY, F. J.; HORVATH, P.; ALMEIDA, R.; MERRILL, W.; DOUGLAS, J.; TAYLOR, J. F. N.; LEVAL, M. R.; STARK, J. - Evaluation of long-term results of homograft and heterograft valves in extracardiac conduits. J. Thorac. Cardiovasc. Surg., 94: 12-19, 1987.

12 CHUN, P. K. C.; ROCCHINI, A. P.; GIBBS, H. R.; ROBINOWITZ, M.; GREEN, D.; VIRMANI, R. - Pannus formation in a Hancock-valved conduit resulting in proximal intraconduit obstruction: late complication of Rastelli procedure for complete transposition of the great vessels with ventricular septal defect and pulmonic stenosis. Am. Heart J., 101: 855-857, 1981.

13 CiARAVElla, J. M.; McGoON, D. C.; DANiELson, G. K.; WALLACE, R. B.; MAIR, D. D. - Experience with the extracardiac conduit. J. Thorac. Cardiovasc. Surg., 78: 920-930, 1979.

14 CLEVELAND, D. C.; KIRKLIN, J. K.; NAFTEL, D. C.; KIRKLIN, J. W.; BLACKSTONE, E. H.; PACÍFICO, A. D.; BARGERON, L. M. - Surgical treatment of tricuspid atresia. Ann. Thorac. Surg., 38: 447-457, 1984.

15 CRAWFORD Jr, F. A.; SADE, R. M.; SPIRALE, F. - Bovine pericardium for conection of congenital heart defects. Ann. Thorac. Surg., 41: 602-605, 1986.

16 DeLEON, S. Y.; IDRISS, F. S.; ILBAWI, M. N.; RASTEGAR, H.; MUSTER, A. J.; PAUL, M. M. - Neointimal obstruction of Carpentiers-Edwards valved conduit in two patients with modified Fontan procedure. Ann. Thorac. Surg., 34: 586-589, 1982.

17 FIORE, A. C.; PEIGH, P. S.; ROBINSON, R. J.; GLANT, M. D.; KING, H.; BROWN, J. W. - Valved and nonvalved right ventricular-pulmonary arterial extracardiac conduits: an experimental comparison. J. Thorac. Cardiovasc. Surg., 86: 490-497, 1983.
18 HAVERICH, A. \& BORST, H. G. - Febrin glue for treatment of bleeding in cardiac surgery. In: BIRCKS, W.; OSTERMEYER, J.; SCHULTE, H. D. (eds.). Cardiovascular surgery. New York, Springer-Verlag, 1980. p. 621-635.

19 HELSETH, H. K.; HAGLIN, J. J.; MONSON, B. K.; WECKSTROM, P. H. - Results of composite graft replacement for aortic root aneurysms. J. Thorac. Cardiovasc. Surg., 80: 754-759, 1980.

IONESCU, M. I. \& DEAC, R. C. - Fascia lata composite graft for right ventricular outflow tract and pulmonary artery reconstruction. Thorax, 25: 427-431, 1970.

21 JONAS, R. A.; FREED, M. D.; MOYER Jr., J. E.; CASTANEEDA, A. R. - Long-term follow-up of patients with synthetic right heart conduits. Circulation, 72: (Parte 2): $77-83,1985$.

JONAS, R. A.; ZIEMER, G.; BRITTON, L.; ARMIGER, L. C. - Cryopreserved and fresh antibiotic-sterilized valved aortic homograft conduits in a long-term sheep model. J. Thorac. Cardiovasc. Surg., 96: 746-755, 1988.

JONES, M.; EIDBO, E. E.; HILBERT, S. L.; FERRANS, V. I.; CLARK, R. E. - The effects of anticalcification treatments on bioprosthetic heart valves implanted in sheep. Trans. Am. Artif. Intern. Organs., 34: 1027-1030, 1988.

24 KOUCHOUKOS, N. T.; MARSHALL, W. G.; WEDIGE-STECHER, T. A. - Eleven-year experience with composite graft replacement of the ascending aorta and aortic valve. J. Thorac. Cardiovasc. Surg., 92: 691-705, 1986.

25 LEVY, R. J.; SCHVEN, F. J.; LUND, S. A.; SMITH, M. S. - Preservation of leaflet calcification of bioprosthetic heart valves with diphosphonate infection therapy. $J$. Thorac. Cardiovasc. Surg., 94: 551-557, 1987.

26 MAIR, D. D.; FULTON, R. E.; JAMIELSON, G. K. - Thrombotic occlusion of Hancock conduit due to severe dehydratation after Fontan operation. Mayo Clin. Proc., 53: $397-400,1978$.

27 MARVASTI, M. A.; PARKER, F. B.; RANDALL, P. A.; WITWER, G. A. - Composite graft replacement of the ascending aorta and aortic valve. J. Thorac. Cardiovasc. Surg., 95: 924-928, 1988.

28 MAZZERA, E.; CORNO, A.; DIDONATO, R.; BELLERINI L.; MARINO, B.; CATENA, G.; MARCELLETTI, C. Surgical bypass of the systemic atrioventricular valve in children by means of a valved conduit. J. Thorac. Cardiovasc. Surg., 96: 321-325, 1988. R. B.; DANIELSON, G. K.; MCGOON, D. C. - Aortic homograft obstruction. J. Thorac. Cardiovasc. Surg., 72: 553-561, 1976. 
SANTOS, J. L. V.; BRAILE, D. M.; SOARES, M. J. F.; RADE, W.; ROSSI, M. A.; THEVENARD, R.; SOUZA, D. R. S. - Avaliaçāo de tubo valvulado de pericárdio bovino em um modelo experimental animal. Rev. Bras. Cir. Cardiovasc., 5(1): 16-25, 1990.

MOON, B. C.;WILLIAMS, W. G.; TRUSLER, G. T.; COLES, J. G.; McLAUGHLIN, P. R. - Re-operation in patients with cryopreserved homograft valved conduits. Circulation, 80 (Supl. 2): 468, 1989.

31 MORAES, C. R. \& RODRIGUES, J. V. - Conduto valvulado de pericárdio bovino: relato de caso. Arq. Bras. Cardiol., 48: 173-177, 1987.

32 PETITE, H.; RINALDI, S.; MENASCHE, P.; PIWNICA, A.; HUC, A. - Low level of calcification mechanical properties of the acyl azide treated pericardium compared with glutaraldehyde treated pericardium. International Symposium on Surgery for Heart Valve Disease, London, Junho 1989. (Resumos).

33 PRENGER, K. B.; HESS, J.; CROMME-DIJKHUIS, A. H.; EJGELAAR, A. - Porcine-valved Dacron conduits in Fontan procedures. Ann. Thorac. Surg., 46: 526-530, 1988.

34 RASTELLI, G. C.; ONGLEY, A.; DAVIS, G. D.; KIRKLIN, J. W. - Surgical repair for pulmonary valve atresia with coronary-pulmonary artery fistula: report of a case. Mayo Clin. Proc., 40: 521-527, 1965.

ROSS, D. N. \& SOMMERVILLE, J. - Conection of pulmonary atresia with a homograft aortic valve. Lancet, 2: 1446-1447, 1966.

36 ROSSI, M. A.; TEIXEIRA, M. D. R.; SOUZA, D. R. S.; PERES, L. C. - Lipid extraction attenuates the calcific degeneration of bovine pericardium used in cardiac valve bioprostheses. (No prelo). Br. J. Exp. Pathol., 1990).

37 SARAVALLI, O. A.; SOMMERVILLE, J.; JEFFERSON, K. E. - Calcification of aortic homograft used for reconstruction of the right ventricle outflow. J. Thorac. Cardiovasc. Surg., 80: 909-912, 1980.

38 SCHLICHTER, A. J. \& KREUTZER, G. O. - Autologous pericardial valved conduit (APVC). Rev. Lat. Cardiol. Circ. Cardiovasc. Infantil, 1: 43-48, 1985.

39 TSAO, J. W.; SHOEN, F. J.; SHANKAR, R.; SALLIS, J. D.; LEVY, R. J. - Retardation of calcification of bovine pericardium used in bioprosthetic heart valves by phosphocitrate and a synthetic analogue. Annual Meeting of the Society for Biomaterials, 13. Junho, 1987 (Resumos).

40 WEBB, C. L.; BENEDICT, J. J.; SCHOEN, F. J.; LINDEN, J. A.; LEVY, R. J. - Inhibition of bioprosthetic heart valve calcification with aminodiphosphonate covalently bound to residual aldehyde groups. Ann. Thorac. Surg., 46: 309-316, 1988.

AGRADECIMENTO: Este trabalho foi desenvolvido com a colaboração da D.P.P. (Divisão de Pesquisas e Publicaçōes) da IMC - Biomédica de São José do Rio Preto.

\section{Discussão}

\section{DR. GERALDO MARTINS RAMALHO Niteró, RJ}

Agradeço à Comissāo Organizadora a indicação do meu nome para comentar este trabalho. Quero enfatizar a importância e a qualidade do trabalho apresentado, principalmente por se tratar de estudo experimental e de assunto ainda controverso. Gostaria de tecer alguns comentários sobre o modelo experimental utilizado, onde o autor emprega cinco ovelhas pesando de 27 a 35 kg e não diz a idade dos animais. Para melhor avaliação das alteraçōes observadas com relaçăo à calcificação ao nível do tubo e da válvula de pericárdio bovino, talvez devessem ser empregados animais de baixa idade, tentando, com isso, reproduzir o que ocorre nas crianças onde empregamos esses tubos valvulados, mais freqüentemente. Com relação às condições hemodinâmicas, gostaria de lembar que a hiper-resistência pulmonar e a má anatomia das artérias pulmonares encontrada nas cardiopatias congênitas modificam, significativamente, a evoluçăo a médio e longo prazo dos tubos valvulados entre o ventrículo direito e a artéria pulmonar. Talvez fosse válido, através de embolização do sistema arterial pulmonar e de cerclagem dos ramos da artéria pulmonar, tentar aproximar o modelo experimental ao observado nas cardiopatias congênitas. Com relação aos resultados, o autor observou gradientes entre 15 e $17 \mathrm{mmHg}$, sendo este último observado a nível da anastomose proximal. Gostaria de perguntar se não seria válido, para evitar essa falha técnica, empregar um anel de sustentação de polipropileno ou outro material ao nível da anastomose proximal, principalmente considerando que essas anastomoses săo realizadas, freqüentemente, em ventrículo direitos hipertrofiados encontrados nas cardiopatias congênitas. Com relação à deposição de cálcio nas cúspides observada na recuperação dos tubos, o autor considerou como "pequena deposição de cálcio". Năo lhe parece pequeno o período de evolução de 6 meses para melhor observar esse fenômeno? $\mathrm{Na}$ discussão, o autor se diz preocupado com a possível angulação do tubo, seu tamanho, tendo causado obstruçăo na anastomose proximal. Gostaria de fazer um comentário e uma sugestão. Considerando que, nas cardiopatias congênitas, freqüentemente o espaço para acomodação do tubo é pequeno, o que poderá causar também obstrução do enxerto, sugeriria, baseado em trabaIho do grupo de Indianópolis, citado na bibliografia, empregar o tubo do pericárdio sustentado externamente por anéis de polipropileno, ou outro material adequado. Concluindo, gostaria de dizer que concordamos com o emprego de tubos de pericárdio bovino, valvulados ou nāo, com opçāo para conexão entre o ventrículo direito e o tronco pulmonar, em casos especiais, após melhor avaliação experimental. Quero, uma vez mais, parabenizar os autores pela qualidade do trabalho, sua apresentação e sua orientação, que segue, perfeitamente, as normas das publicaçōes científicas. Obrigado. 
SANTOS, J. L. V.; BRAILE, D. M.; SOARES, M. J. F.; RADE, W.; ROSSI, M. A.; THEVENARD, R.; SOUZA, D. R. S. - Avaliação de tubo valvulado de pericárdio bovino em um modelo experimental animal. Rev. Bras. Cir. Cardiovasc., 5(1): 16-25, 1990.

\section{DR. GILBERTO V. BARBOSA Porto Alegre, RS}

Este trabalho provém de uma Instituição séria e responsável, com tradição em pesquisa aplicada e pioneira na utilização do pericárdio bovino em cirurgia cardiovascular, desde 1979, e eu me sinto honrado em comentá-lo. A cirurgia de conexão do ventrículo venoso ao tronco pulmonar através de conduto valvulado extracardíaco, praticada de rotina nos últimos 25 anos, tornou oportuna a análise tardia e a seleção daqueles condutos de melhor desempenho. As dificuldades de obtenção e armazenamento dos homoenxertos de aorta e a freqüente necessidade de extendê-los com tecido de $\mathrm{Da}$ cron, apesar de sua razoável performance, restringem o seu uso no nosso meio. Nos heteroenxertos de Dacron com válvula biológica, os inconvenientes estão na formação exagerada da neoíntima e na degeneração fibrocálcica da válvula biológica, provocando obstruçōes e a necessidade de reintervenção de $30 \%$ em 5 anos. A análise desses fatos justifica a tentativa de encontrar alternativas mais confiáveis e de maior durabilidade. A confecção, utilizaçāo clínica e disponibilidade comercial de condutos de pericárdio bovino com valva sem suporte foi descrita por Moraes, em 87 e 88 , relatando bons resultados a curto prazo. $\mathrm{O}$ conduto do IMC, além das vantagens já relatadas, tem custo acessível e não necessita alongamento com tecidos sintéticos, sendo, portanto, um monoenxerto. Propicia, ainda, pouca aderência às estruturas mediastinais responsáveis nas reintervençōes dos outros condutos por $25 \%$ de laceraçōes, com alta mortalidade. A válvula é montada em suporte rígido com hastes, o que propicia, nos condutos com diâmetros inferiores a $18 \mathrm{~mm}$, serem potencialmente estenóticos. Nos trabalhos internacionais, ficou demonstrado que 0 estresse hemodinâmico provoca fibrose mais acentuada na porção proximal do conduto e nos componentes da válvula, advindo, depois, por múltiplos fatores, a calcificação. Disto resulta obstrução progressiva mais acentuada nas crianças e adultos jovens, principalmente clientela desses enxertos, obrigando a reintervenção em $56 \%$ dos casos, em 10 anos. Neste trabalho, os exames macroscópico e histológico mostraram alteraçōes degenerativas discretas sem comprometimento estrutural e funcional do enxerto - o que é ótimo, mas para estarmos seguros da manutenção desse excepcional desempenho, teremos que avaliá-lo numa série clínica de cinco a 10 anos, pois os três objetivos básicos da sua pesquisa são tempo-dependentes. Não foram mencionados, no trabalho escrito, os diâmetros dos tubos utilizados e nem o seu comprimento. Seria conveniente aparecer, na Tabela 1 ou noutra, o resultado do gradiente sistólico de pico entre o ventrículo direito $e$ a artéria pulmonar, obtidos no cateterismo. Com os dados da Tabela 1, calculamos as médias dos gradientes obtidos por ecodopplercardiografia para comparação entre os dois e seis meses, obtendo os valores de 16 e $33 \mathrm{mmHg}$, respectivamente, mostrando um aumento de $17 \mathrm{mmHg}(100 \%)$, no período de observação. Excluidas as estenoses técnicas nas áreas de anastomose, é possível que as alteraçōes degenerativas descritas nos estudos histológico e macroscópico tenham relação com a elevação desses gradientes. Na ótima bibliografia, seria útil incluir os trabalhos pertinentes de João Bosco de Oliveira e col., de junho de 86, Carlos Roberto Moraes e col., e de Rui Sequeira de Almeida, de agosto de 88 , publicados nas revistas brasileiras. Esta pesquisa, bem planejada e elegantemente executada, tem, ainda, o mérito de colocar à disposição da comunidade de cirurgiōes um novo conduto para uso clínico. Eu felicito o autor pela excelente apresentação. Obrigado.

\section{DR. VERDE DOS SANTOS (Encerrando)}

Gostaria de agradecer as palavras amáveis dos Drs. Gilberto e Geraldo e dizer que suas críticas construtivas serão incluídas no texto original visando à publicação. Respondendo à pergunta do Dr. Giberto, nós utilizamos tubo de $21 \mathrm{~mm}$ de diâmetro (com bioprótese de pericárdio bovino n: 21) em dois casos e de $23 \mathrm{~mm}$ nos outros três casos. $\mathrm{O}$ aumento dos gradientes medidos por ecodopplercardiografia aumentam no sexto mês em relação ao terceiro, como já era esperado, porque os animais cresceram. Portanto, acreditamos que os pontos de calcificação encontrados nas biopróteses sem alterar o funcionamento das mesmas não foram os responsáveis isolados pelo aumento dos gradientes. Ao Dr. Geraldo, respondo que a idade dos animais variou de 4 a 6 meses, sendo que o animal de $28 \mathrm{~kg}$ tinha 4 meses, com o peso aumentando em média de $25 \%$ em 6 meses. 0 tempo de seguimento escolhido foi de 6 meses, porque isso corresponde a 5 ou 6 anos no humano, e as alteraçōes ocorridas no enxerto não foram tão importantes, - que nos leva a crer que duraria muito mais tempo, se fosse permitido. Para finalizar, quero agradecer ao Dr. Braile, ao Dr. Ardito e à bióloga Dorotéia pelo incentivo e participação direta na confecção deste trabalho. 